\title{
Magnetic and charge susceptibilities in the half-filled triangular lattice Hubbard model
}

\author{
Shaozhi Li $\odot^{1}$ and Emanuel Gull $\odot^{1,2}$ \\ ${ }^{1}$ Department of Physics, University of Michigan, Ann Arbor, Michigan 48109, USA \\ ${ }^{2}$ Center for Computational Quantum Physics, The Flatiron Institute, New York, New York 10010, USA
}

(Received 22 August 2019; revised manuscript received 27 January 2020; accepted 21 February 2020; published 11 March 2020)

\begin{abstract}
We study the momentum-dependent magnetic and charge susceptibilities in the half-filled two-dimensional triangular Hubbard model within the ladder dual fermion approximation in the metallic, Mott insulating, and crossover regions of parameter space. In the insulating state, we find strong spin fluctuations at the $K$ point at low energy corresponding to the $120^{\circ}$ antiferromagnetic order. The spin spectra of the insulating state is consistent with the inelastic neutron spectra of the triangular compound $\mathrm{Ba}_{8} \mathrm{CoNb}_{6} \mathrm{O}_{24}$. We also find that the spin fluctuations at the $K$ point persist into the metallic phase and move to higher energy. These results are in agreement with studies of spin-lattice relaxation times in $\kappa-(\mathrm{ET})_{2} \mathrm{Cu}_{2}(\mathrm{CN})_{3}$. Finally, we present charge susceptibilities in different areas of parameter space, which should correspond to momentum-resolved electron-loss spectroscopy measurements on triangular compounds.
\end{abstract}

DOI: 10.1103/PhysRevResearch.2.013295

\section{INTRODUCTION}

Experimental evidence on several organic materials, including $\kappa$-(BEDT-TTF $)_{2} \mathrm{Cu}_{2}(\mathrm{CN})_{3} \quad[1,2], \mathrm{EtMe}_{3} \mathrm{Sb}[\mathrm{Pd}$ $\left.(\mathrm{dmit})_{2}\right]_{2}$ [3-5], and $\kappa-\mathrm{H}_{3}(\mathrm{Cat}-\mathrm{EDT}-\mathrm{TTF})_{2}$ [6], suggests that these compounds are close to a two-dimensional triangular structure and exhibit interesting electron correlation behaviors including, potentially, a quantum spin-liquid phase [7] in the ground state [8]. These compounds, as well as the low-energy physics of the fully isotropic triangular material $\mathrm{Ba}_{8} \mathrm{CoNb}_{6} \mathrm{O}_{24}$ [9], may be described by a half-filled singleorbital Hubbard model on a triangular two-dimensional lattice, with an on-site Coulomb interaction strength comparable to or larger than the bandwidth [10].

Because of the subtle competition of metallic, ordered, and spin-liquid phases in the ground state $[11,12]$, this model has been studied extensively with a wide range of numerical tools, including exact diagonalization (ED) [13-15], density matrix renormalization group theory (DMRG) [8], variational Monte Carlo (VMC) [16-19], variational cluster approximation [20-22], strong-coupling expansions [23], path-integral renormalization group techniques [24], and cluster dynamical mean-field theory (DMFT) in the cellular [25-29] and dynamical cluster [30,31] variants. The focus in most of these studies has been on the precise location of the phase boundaries, ordering (or the absence thereof), and on the nature of these phases.

Experimentally, much of our knowledge about correlated triangular systems is obtained from single- and two-particle scattering experiments such as photoemission [32], Raman

Published by the American Physical Society under the terms of the Creative Commons Attribution 4.0 International license. Further distribution of this work must maintain attribution to the author(s) and the published article's title, journal citation, and DOI. spectroscopy [33], nuclear magnetic resonance (NMR) [2,34], or inelastic neutron scattering (INS) [9,35]. To understand these experimental results, it is necessary to calculate the corresponding response functions as a function of energy and momentum. For neutron spectroscopy and angular-resolved photoemission spectroscopy, in particular, both fine momentum and energy resolutions are desired. Such results are difficult to obtain, as computational methods formulated on finite lattices (such as ED, DMRG, and cluster DMFT) provide limited momentum resolution. In addition, quantum Monte Carlo approaches are impeded by a sign problem in frustrated systems [36]. Results for these quantities are therefore often obtained from fits to quantum spin models, which are only justified in the large Coulomb interaction limit.

In this paper, we provide results for the momentum and energy dependence of the spin and charge spectra of the half-filled triangular lattice Hubbard model. We use the ladder dual fermion approximation (LDFA), which is a diagrammatic extension of the DMFT and recovers continuous momentum dependence without a sign problem. We perform simulations from strong to weak interactions and systematically study spin and charge spectra in different areas of parameter space. We find that strong spin fluctuations at the $K$ point at low energy, corresponding to the $120^{\circ}$ antiferromagnetic order, persist into the metallic phase. These results are also consistent with experimental NMR observations.

\section{MODEL AND METHODOLOGY}

The Hubbard model is defined as

$$
H=-t \sum_{\langle i j\rangle, \sigma}\left(c_{i \sigma}^{\dagger} c_{j \sigma}+\text { H.c. }\right)+U \sum_{i} \hat{n}_{i \uparrow} \hat{n}_{i \downarrow}-\mu \sum_{i, \sigma} \hat{n}_{i \sigma} .
$$

$\langle\cdots\rangle$ denotes a summation over nearest neighbors; $c_{i \sigma}^{\dagger}\left(c_{i \sigma}\right)$ creates (annihilates) an electron with spin $\sigma$ on site $i$; $\hat{n}_{i \sigma}=$ $c_{i \sigma}^{\dagger} c_{i \sigma}$ is the particle number operator; $U$ is the on-site 
Coulomb interaction; $t$ is the nearest-neighbor hopping integral; and $\mu$ is the chemical potential. We set $t=1$ throughout this paper and restrict ourselves to half filling.

\section{A. Ladder dual fermion approximation}

We study the model in the ladder dual fermion approximation [37-42] using the open source code of Ref. [43]. The dual fermion technique is an exact mapping that transforms the lattice system into a computationally tractable impurity model and correction terms. The LDFA is an approximation to this exact scheme that uses a nonperturbative DMFT impurity model as a starting point [44] and perturbatively adds nonlocal spin and charge correlations $[42,43]$. Therefore, LDFA recovers some (but not all) of the nonlocal correlations. DMFT calculations are performed with the continuous time auxiliary field quantum Monte Carlo method $[45,46]$ with submatrix updates [47]. LDFA is accurate at high temperature [48] but uncontrolled in practice in the sense that adding systematic corrections, while possible in theory [49,50], is not feasible for the parameters studied here. A detailed assessment of the approximation errors of the susceptibility and the singleparticle properties on the square lattice [51] showed that while doping- and interaction-dependent scaling effects were present, the overall momentum dependence was accurate.

In the following, we repeat the formulas of the LDFA [37-39]. The action for the Hubbard model is

$$
\begin{aligned}
S\left[c^{*}, c\right]= & -\sum_{\omega, \mathbf{k}, \sigma} c_{\omega, \mathbf{k}, \sigma}^{*}\left[i \omega+\mu-h_{\mathbf{k}}\right] c_{\omega, \mathbf{k}, \sigma} \\
& +\sum_{i} U \int_{0}^{\beta} c_{i, \uparrow}^{*}(\tau) c_{i, \uparrow}(\tau) c_{i, \downarrow}^{*}(\tau) c_{i, \downarrow}(\tau) \mathcal{D}\left[\mathbf{c}^{*}, \mathbf{c}\right],
\end{aligned}
$$

where $c_{i, \sigma}^{*}(\tau)$ and $c_{i, \sigma}(\tau)$ are Grassmann numbers corresponding to creation and annihilation operators at imaginary time $\tau$ and site $i ; c_{\omega, \mathbf{k}, \sigma}^{*}\left(c_{\omega, \mathbf{k}, \sigma}\right)$ is the Grassmann number at the momentum $\mathbf{k}$ and fermion Matsubara frequency $\omega . h_{\mathbf{k}}$ is the bare dispersion and $\beta$ is the inverse temperature $\beta=1 / T$.

In the spirit of the DMFT, we introduce a single-site reference system and rewrite the lattice action (1) as

$$
\begin{aligned}
S\left[c^{*}, c\right]= & \sum_{i} S_{\mathrm{imp}}\left[c_{i}^{*}, c_{i}\right]-\sum_{\omega k \sigma}\left(\Delta_{\omega}-h_{k}\right) c_{\omega k \sigma}^{*} c_{\omega k \sigma} \\
S_{\mathrm{imp}}\left[c_{i}^{*}, c_{i}\right]= & -\sum_{\omega, \mathbf{k}, \sigma} c_{\omega, \mathbf{k}, \sigma}^{*}\left[i \omega+\mu-\Delta_{\omega}\right] c_{\omega, \mathbf{k}, \sigma} \\
& +\sum_{i} U \int_{0}^{\beta} c_{i, \uparrow}^{*}(\tau) c_{i, \uparrow}(\tau) c_{i, \downarrow}^{*}(\tau) \\
& \times c_{i, \downarrow}(\tau) \mathcal{D}\left[\mathbf{c}^{*}, \mathbf{c}\right],
\end{aligned}
$$

where $\Delta_{\omega}$ is a hybridization function. The DF formalism [37-39] proceeds by introducing new dual degrees of freedom $f^{*}, f$ via a Hubbard-Stratonovich transformation of the second term on the right-hand side of Eq. (2) and integrating out the original degrees of freedom $c^{*}, c$. In this way, the action (2) is divided into two separate problems: an impurity action and a dual action

$$
\tilde{S}\left[f^{*}, f\right]=-\sum_{\mathbf{k}, \omega, \sigma} f_{\mathbf{k} \omega \sigma}^{*}\left(\tilde{G}_{\mathbf{k} \omega \sigma}^{(0)}\right)^{-1} f_{\mathbf{k} \omega \sigma}+\tilde{V}\left[f^{*}, f\right],
$$

where $\tilde{G}_{\mathbf{k} \omega \sigma}^{(0)}=\left[g_{\omega}^{-1}+\Delta_{\omega}-h_{\mathbf{k}}\right]^{-1}-g_{\omega}$, and $g_{\omega}$ is the impurity Green's function. The dual interaction is given by $\tilde{V}\left[f^{*}, f\right]=-\frac{1}{4} \gamma_{\alpha \beta \gamma \delta} f_{\alpha}^{*} f_{\beta} f_{\gamma}^{*} f_{\delta}+\cdots$, where small Greek letters $(\alpha, \beta, \gamma$, and $\delta)$ stand for the set $\{\omega, \mathbf{k}, \sigma\}$,

$$
\gamma_{\alpha \beta \gamma \delta}=g_{\alpha \alpha^{\prime}}^{-1} g_{\gamma \gamma^{\prime}}^{-1}\left[g_{\alpha^{\prime} \beta^{\prime} \gamma^{\prime} \delta^{\prime}}^{\mathrm{imp}(4)}-g_{\alpha^{\prime} \beta^{\prime}} g_{\gamma^{\prime} \delta^{\prime}}+g_{\alpha^{\prime} \delta^{\prime}} g_{\gamma^{\prime} \beta^{\prime}}\right] g_{\beta^{\prime} \beta}^{-1} g_{\delta^{\prime} \delta}^{-1},
$$

and the two-particle Green's function of the impurity site is defined as

$$
g_{\alpha \beta \gamma \delta}^{\operatorname{imp}(4)}=\frac{1}{Z} \int c_{\alpha} c_{\beta} c_{\gamma}^{*} c_{\delta}^{*} \exp \left(-S\left[\mathbf{c}^{*}, \mathbf{c}\right]\right) \mathcal{D}\left[\mathbf{c}^{*}, \mathbf{c}\right] .
$$

In LDFA, only the ladder series of the first term of the dual interaction $\tilde{V}\left[f^{*}, f\right]$ is considered. This leads to a set of coupled equations that relate the charge/magnetic vertices, the dual self-energy $\tilde{\Sigma}$, and the dual Green's function to each other. The self-energy of dual fermions is defined as

$$
\begin{aligned}
\tilde{\Sigma}_{\omega \mathbf{k} \sigma}= & \frac{T}{2 N} \sum_{\nu \mathbf{q}}\left(3\left[\Gamma_{\omega \omega \nu}^{m}(\mathbf{q})-\frac{1}{2} \Gamma_{\omega \omega}^{(2), m}(\mathbf{q} v)\right]\right. \\
& \left.+\Gamma_{\omega \omega \nu}^{d}(\mathbf{q})-\frac{1}{2} \Gamma_{\omega \omega \nu}^{(2), d}(\mathbf{q})\right) \tilde{G}_{\omega \mathbf{k}+\mathbf{q} \sigma},
\end{aligned}
$$

where $v$ is the bosonic Matsubara frequency and $\mathbf{q}$ is the momentum. $d$ and $m$ represent the charge and magnetic channels. Equation (6) relies on the second-order vertices,

$$
\Gamma_{\omega \omega^{\prime} v}^{(2), d / m}=\gamma_{\omega \omega^{\prime \prime} \nu}^{d / m} \tilde{\chi}_{\omega^{\prime \prime} v}^{0}(\mathbf{q}) \gamma_{\omega^{\prime \prime} \omega^{\prime} v}^{d / m},
$$

where $\omega^{\prime}$ and $\omega^{\prime \prime}$ are fermion Matsubara frequencies, $\tilde{\chi}_{\omega \nu}^{0}=$ $-\frac{T}{N} \sum_{\mathbf{k}} \tilde{G}_{\omega \mathbf{k} \sigma} \tilde{G}_{\omega+\nu \mathbf{k}+\mathbf{q} \sigma}$, and the fully dressed vertex function

$$
\Gamma_{\omega \omega^{\prime} v}^{d / m}(\mathbf{q})=\gamma_{\omega \omega^{\prime} v}^{d / m}+\sum_{\omega^{\prime \prime}} \gamma_{\omega \omega^{\prime \prime} v}^{d / m} \tilde{\chi}_{\omega^{\prime \prime} v}^{0}(\mathbf{q}) \Gamma_{\omega^{\prime \prime} \omega^{\prime} v}^{d / m}(\mathbf{q}) .
$$

The dual Green's function is computed by using the Dyson equation

$$
\tilde{G}_{\omega \mathbf{k} \sigma}^{-1}=\left[\tilde{G}_{\omega \mathbf{k} \sigma}^{(0)}\right]^{-1}-\tilde{\Sigma}_{\omega \mathbf{k} \sigma} .
$$

Equations (6) and (9) are iterated self-consistently until convergence of $\tilde{G}$ is achieved. At convergence, the lattice Green's function is evaluated as

$$
\begin{aligned}
G_{\omega \mathbf{k} \sigma}^{-1}= & {\left[1+\tilde{\Sigma}_{\omega \mathbf{k} \sigma} g_{\omega \sigma}\right]^{-1}\left[g_{\omega \mathbf{k}}^{-1}+\left(\Delta_{\omega}-h_{\mathbf{k}}\right)\right.} \\
& \left.+\tilde{\Sigma}_{\omega \mathbf{k} \sigma} g_{\omega \sigma}\left(\Delta_{\omega}-h_{\mathbf{k}}\right)\right] .
\end{aligned}
$$

The hybridization function in Eq. (2) is not known a priori and needs to be found self-consistently. In our calculations, we start from the hybridization function of DMFT and calculate the lattice Green's function using the LDFA approach. We then update the hybridization function as

$$
\Delta_{\text {new }}=\Delta_{\text {old }}+\xi\left[g^{-1}-G_{\text {loc }}^{-1}\right],
$$

where $G_{\text {loc }}$ is the local lattice Green's function and $\xi$ is an updating weight. In our calculations, we set $\xi=0.2$ to make the convergence stable. 
After convergence of the hybridization function, the lattice two-particle Green's function $G_{\alpha \beta \gamma \delta}^{(4)}$ is obtained as

$$
\begin{aligned}
& G_{\alpha \beta \gamma \delta}^{(4)}-[G \otimes G]_{\alpha \beta \gamma \delta} \\
& \quad=L_{\alpha \alpha^{\prime}} L_{\beta \beta^{\prime}}\left(\tilde{G}^{(4)}-\tilde{G} \otimes \tilde{G}\right)_{\alpha^{\prime} \beta^{\prime} \gamma^{\prime} \delta^{\prime}} R_{\gamma \gamma^{\prime}} R_{\delta \delta^{\prime}},
\end{aligned}
$$

where $\tilde{G}_{\alpha \beta \gamma \delta}^{(4)}$ is the two-particle Green's function of dual fermions, $(A \otimes B)_{\alpha \beta \gamma \delta}=A_{\alpha \delta} B_{\beta \gamma}-A_{\alpha \gamma} B_{\beta \delta}$, $L_{\alpha \beta}=\left[(\Delta-h)^{-1} g^{-1}\right]_{\alpha \beta}^{-1}, \quad$ and $\quad R_{\alpha \beta}=\left[g^{-1}(\Delta-h)^{-1}\right]_{\alpha \beta}^{-1}$ [38].

The lattice charge susceptibility is defined as

$$
\begin{aligned}
\chi_{d}(\mathbf{q}, i v)= & \frac{1}{\beta^{2} N^{2}} \sum_{\substack{\mathbf{k}, \mathbf{k}^{\prime}, \omega, \omega^{\prime},}}\left\langle c_{\mathbf{k} \omega \uparrow}^{\dagger} c_{\mathbf{k}+\mathbf{q} \omega+\nu \uparrow} c_{\mathbf{k}^{\prime}+\mathbf{q} \omega^{\prime}+v \sigma}^{\dagger} c_{\mathbf{k} \omega^{\prime} \sigma}\right\rangle \\
& -\frac{1}{\beta^{2} N^{2}} \sum_{\mathbf{k}, \mathbf{k}^{\prime}, \omega, \omega^{\prime}} G_{\mathbf{k} \omega \uparrow} G_{\mathbf{k}^{\prime} \omega^{\prime} \sigma} \delta_{\mathbf{q}, 0} \delta_{v, 0} \\
= & \frac{1}{\beta^{2} N^{2}} \sum_{\mathbf{k}, \mathbf{k}^{\prime}, \omega, \omega^{\prime}, \sigma} G_{\mathbf{k} \omega^{\prime} \sigma, \mathbf{k}+\mathbf{q} \omega+\nu \uparrow, \mathbf{k} \omega \uparrow, \mathbf{k}^{\prime}+\mathbf{q} \omega^{\prime}+v \sigma}^{(4)} \\
& -\frac{1}{\beta^{2} N^{2}} \sum_{\mathbf{k}, \mathbf{k}^{\prime}, \omega, \omega^{\prime}} G_{\mathbf{k} \omega \uparrow} G_{\mathbf{k}^{\prime} \omega^{\prime} \sigma} \delta_{\mathbf{q}, 0} \delta_{v, 0},
\end{aligned}
$$

and the magnetic susceptibility is defined as

$$
\begin{aligned}
\chi_{m}(\mathbf{q}, i v) & =\frac{1}{2 \beta^{2} N^{2}} \sum_{\substack{\mathbf{k}, \mathbf{k}^{\prime}, \omega, \omega^{\prime}, \sigma}} x_{\sigma}\left\langle c_{\mathbf{k} \omega \uparrow}^{\dagger} c_{\mathbf{k}+\mathbf{q} \omega+\nu \uparrow} c_{\mathbf{k}^{\prime}+\mathbf{q} \omega^{\prime}+v \sigma}^{\dagger} c_{\mathbf{k} \omega^{\prime} \sigma}\right\rangle \\
& =\frac{1}{2 \beta^{2} N^{2}} \sum_{\substack{\mathbf{k}, \mathbf{k}^{\prime}, \omega, \omega^{\prime},}} x_{\sigma} G_{\mathbf{k} \omega^{\prime} \sigma, \mathbf{k}+\mathbf{q} \omega+\nu \uparrow, \mathbf{k} \omega \uparrow, \mathbf{k}^{\prime}+\mathbf{q} \omega^{\prime}+v \sigma}^{(4)},
\end{aligned}
$$

where $x_{\sigma}=1(-1)$ as $\sigma=\uparrow(\downarrow)$, and $N$ is the number of lattice sites.

LDFA calculations are performed on a momentum space grid-here, we choose a square $24 \times 24$ cluster, resulting in 288 points in the triangular lattice Brillouin zone. Both the single-particle Green's function and two-particle susceptibilities are defined on that grid.

\section{B. Comparisons of DMFT and LDFA}

To show the importance of nonlocal correlations included in our LDFA approach, we compare the static magnetic susceptibility $\chi_{m}\left(\mathbf{q}, i \nu_{0}\right)$ of dynamical mean-field theory and the ladder dual fermion approach. $\chi_{m}\left(\mathbf{q}, i v_{0}\right)$ of DMFT is given by

$$
\chi_{m}(\mathbf{q}, i v=0)=\frac{1}{2 \beta^{2}} \sum_{\omega, \omega^{\prime}} \chi_{\omega \omega^{\prime} v=0}^{m}(\mathbf{q})
$$

where $\omega$ and $\omega^{\prime}$ are the fermion Matsubara frequencies and $v$ is the bosonic Matsubara frequency. $\chi_{\omega \omega^{\prime} v}^{m}(\mathbf{q})$ is calculated by [52]

$$
\left[\chi_{v}^{m}(\mathbf{q})\right]_{\omega \omega^{\prime}}^{-1}=\left[\chi_{v}^{0}(\mathbf{q})\right]_{\omega \omega^{\prime}}^{-1}+\left[\tilde{\chi}_{v}^{m}(\mathbf{q})\right]_{\omega \omega^{\prime}}^{-1}-\left[\tilde{\chi}_{v}^{0}(\mathbf{q})\right]_{\omega \omega^{\prime}}^{-1},
$$

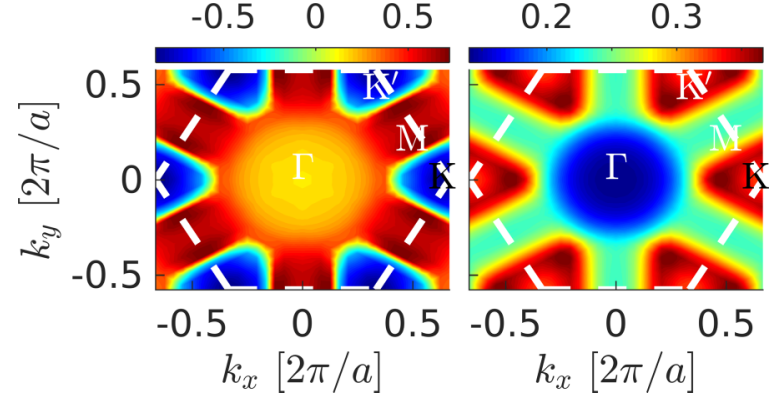

FIG. 1. Static magnetic susceptibility in momentum space $\chi_{m}\left(\mathbf{q}, i v_{0}\right)$ for $U=6 t$ and $T=t / 6$. The left (right) panel shows results of DMFT (LDFA).

where $\left[\tilde{\chi}_{v}^{0}(\mathbf{q})\right]_{\omega \omega^{\prime}}^{-1}$ is the bare bubble of the impurity site, $\left[\tilde{\chi}_{v}^{m}(\mathbf{q})\right]_{\omega \omega^{\prime}}^{-1}$ is the magnetic susceptibility of the impurity site, and $\left[\chi_{\nu}^{0}(\mathbf{q})\right]_{\omega \omega^{\prime}}^{-1}$ is the lattice bare bubble, which is given by $\left[\chi_{v}^{0}(\mathbf{q})\right]_{\omega \omega^{\prime}}^{-1}=-\frac{\beta}{N} \sum_{\mathbf{k}} G(\mathbf{k}, \omega) G(\mathbf{k}+\mathbf{q}, \omega) \delta_{\omega \omega^{\prime}}$.

Figure 1 shows $\chi_{m}\left(\mathbf{q}, i v_{0}\right)$ of DMFT (left panel) and LDFA (right panel) at $U=6 t$ and $\beta=6 / t$. The left panel shows negative values near the $K$ point, indicating a divergence of the vertex at these momentum points. In contrast, the right panel shows that $\chi_{m}\left(\mathbf{q}, i v_{0}\right)$ of LDFA is small, implying that the spin fluctuations are weak. These inconsistencies suggest that DMFT cannot accurately describe the momentum-dependent spin fluctuations in this parameter regime.

\section{Maximum entropy}

To examine spectral properties, we use the ALPS implementation [53,54] of the maximum-entropy method [55] to perform the analytic continuation of Matsubara data to the real frequency space. Analytic continuation is numerically ill posed, and many different spectral functions will yield the same Matsubara response within error bars. To validate our calculations, we compare the imaginary part of the dynamical magnetic susceptibility $\operatorname{Im} \chi_{m}(\mathbf{q}, \omega)$ from the maximum-entropy method to the experimental data obtained from $\mathrm{Ba}_{8} \mathrm{CoNb}_{6} \mathrm{O}_{24}$ [9] in Fig. 2. To the left of the green (a) experiment

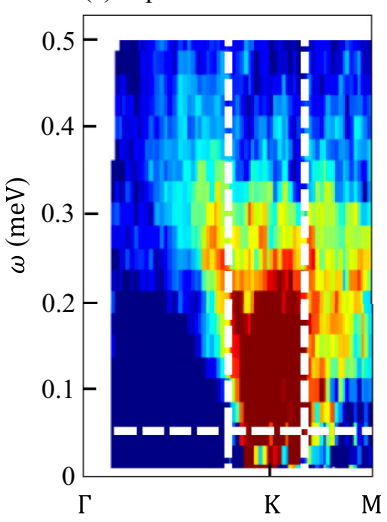

(b) theory

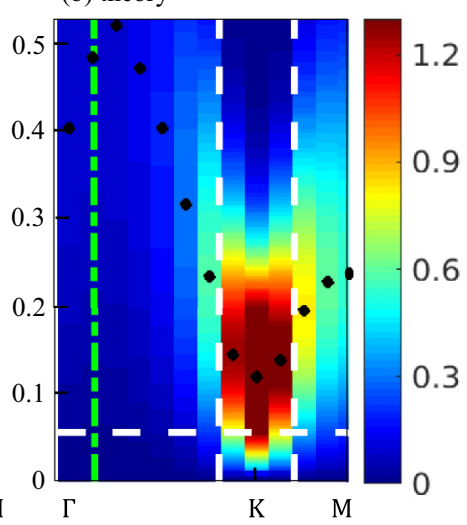

FIG. 2. (a) Spin susceptibility $\operatorname{Im} \chi_{m}(\mathbf{q}, \omega)$ obtained from INS on $\mathrm{Ba}_{8} \mathrm{CoNb}_{6} \mathrm{O}_{24}$ by Rawl et al. [9]. (b) LDFA calculation at $U=12 t$ and $T=t / 6$, using a fit of $t=3 \mathrm{meV}$. The black dots show the location of the maximum strength at each momentum. 


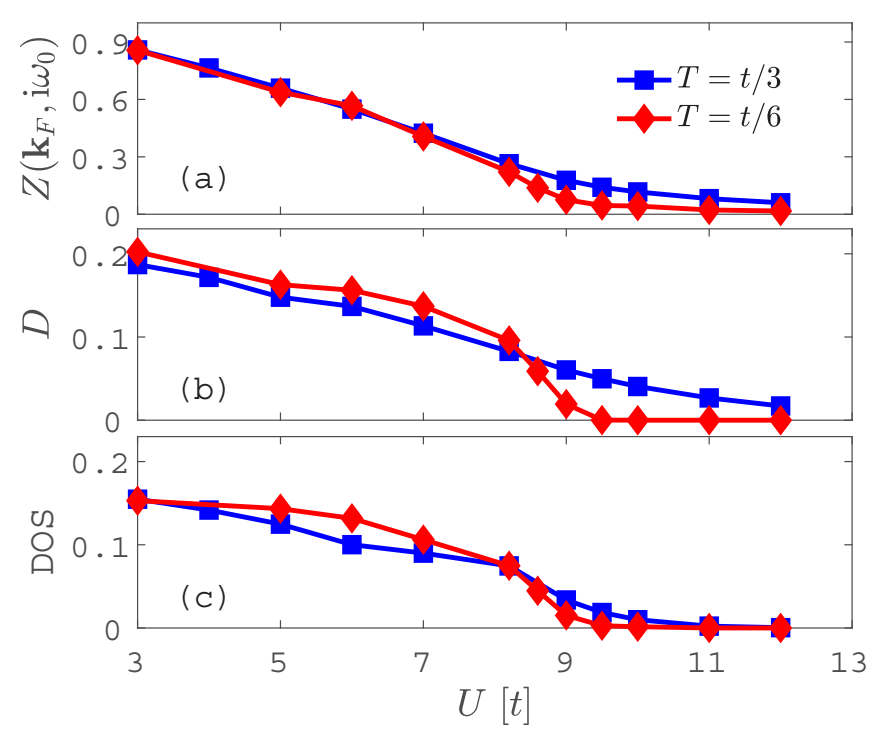

FIG. 3. Metal-insulator crossover as a function of $U$, for temperature $T=t / 3$ and $T=t / 6$. (a) Evolution of the quasiparticle weight estimate $Z$ at momentum $\mathbf{k}_{F}=(5 \pi / 6,0)$. (b) Double occupancy. (c) Local density of states.

line, no experimental data are available. Our simulations were obtained for $U=12 t$ and $T=t / 6$, and we set $t=3 \mathrm{meV}$ to fit the experimental data. Both our numerical data and the experimental data show that the intensity of the spin excitation around the $K$ point is strong. We also note that the spin gap at the $K$ point and the spin excitation energy at the $M$ point are similar. This consistent behavior implies that our magnetic susceptibilities capture the main features of the spin spectra in triangular systems.

\section{RESULTS}

\section{A. Metal-insulator crossover}

The half-filled Hubbard model exhibits a metal-insulator phase transition on the triangular lattice at zero temperature $[8,22]$, which has been widely studied by using LDFA $[30,56,57]$ and other methods [8,22]. In this work, we set the temperature above the critical temperature of the first-order phase transition observed in DMFT studies [30], such that the system exhibits metallic behavior for $U \leqslant 8 t$, crossover behavior for $8 t<U<9.5 t$, and insulating behavior for $U \geqslant 9.5 t$. Figure 3 shows variation of the quasiparticle weight $Z$, the double occupancy $D=\left\langle\hat{n}_{\uparrow} \hat{n}_{\downarrow}\right\rangle$, and the local density of states at temperature $T=t / 3$ and $T=$ $t / 6 . Z$ is approximately determined as $Z\left(\mathbf{k}_{F}, i \omega_{0}\right)=1 /[1-$ $\left.\operatorname{Im} \Sigma\left(\mathbf{k}_{F}, i \omega_{0}\right) / \omega_{0}\right]$, where $\Sigma\left(\mathbf{k}_{F}, i \omega_{0}\right)$ is the self-energy at the lowest Matsubara frequency $\omega_{0}$, and $\mathbf{k}_{F}=(5 \pi / 6 a, 0)$ is a momentum on the Fermi surface of the noninteracting system. The double occupancy is obtained via $D=$ $\frac{1}{2}\left[\chi_{d}^{\text {loc }}(\tau=0)-2 \chi_{m}^{\text {loc }}(\tau=0)+2\left\langle\hat{n}_{\uparrow}\right\rangle\left\langle\hat{n}_{\downarrow}\right\rangle\right]$, where $\chi_{d(s)}^{\text {loc }}(\tau)$ is the local charge (spin) susceptibility. Figure 3(c) plots the local density of states (DOS) at the Fermi surface, obtained via analytic continuation of the local electron Green's function. All three quantities are large at small $U$, consistent with metallic behavior, and approach a small value at a large $U$, consistent with insulating behavior.

To illustrate the behavior in these three regions, we choose one point in each region: $U=6 t$ in the metallic phase, $U=$ $8.2 t$ in the crossover region, and $U=12 t$ in the insulating phase.

\section{B. Spectral function}

Figures 4(a)-4(c) show the momentum-resolved singleparticle spectral functions $A(\mathbf{k}, \omega)$ for $U=6 t, 8.2 t$, and $12 t$ at $T=t / 6$. The white dashed line indicates the Fermi surface. At $U=6 t$, when it is a metal, there is a strong quasiparticle peak at the Fermi surface. At $U=8.2 t$, the intensity of this quasiparticle peak is suppressed and the lower (upper) Hubbard band forms around the $K(\Gamma)$ point. At $U=12 t$, a Mott gap is fully opened. Our calculations show that the Mott gap is opened as $U>10 t$ (see Fig. 3). We note that there is no superstructure along the $\Gamma-K$ direction in Fig. 4(c) as longranged magnetic order is absent. These features are consistent with previous cluster perturbation theory results [22].

\section{Magnetic susceptibility}

Here, we focus on the magnetic properties. Figure 5 shows the momentum-resolved static and dynamical magnetic susceptibility for $U=6 t, U=8.2 t$, and $U=12 t$ at $T=t / 6$. The static magnetic susceptibilities $\chi_{m}\left(\mathbf{q}, i v_{0}=0\right)$ for these three values of $U$ are plotted in Figs. 5(a)- 5(c), respectively. The white dashed line represents the boundary of the Brillouin zone. Static spin correlations are enhanced as $U$ increases. At $U=12 t$, the static spin correlations show a clear peak at the $K$ point, which is much stronger than that in the metallic $(U=6 t)$ and crossover $(U=8.2 t)$ regions. The strong peak at $U=12 t$ indicates the formation of $120^{\circ}$ antiferromagnetic (AFM) spin fluctuations [8], which will magnetically order at low temperature $[8,27,58]$.
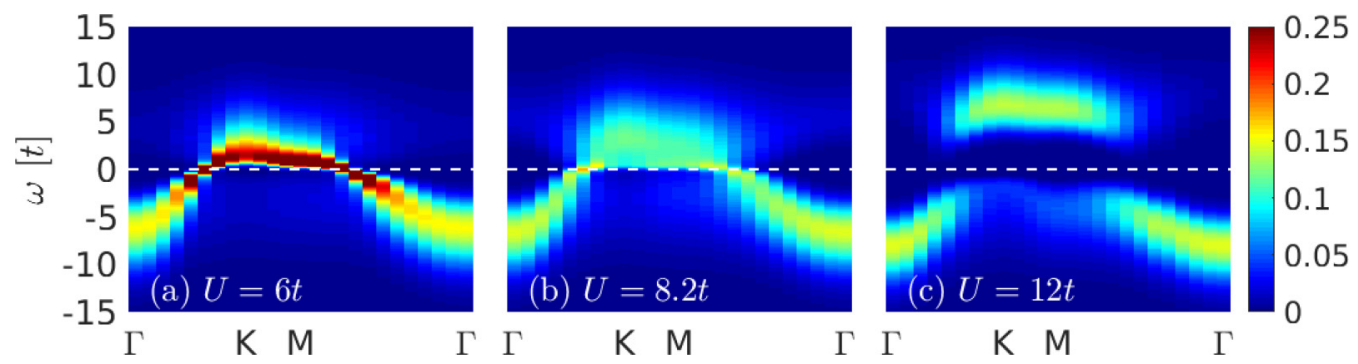

FIG. 4. Momentum dependence of the spectral function $A(\mathbf{k}, \omega)$ for (a) $U=6 t$, (b) $U=8.2 t$, and (c) $U=12 t$ at $T=t / 6$. The white dashed line shows the Fermi surface. 

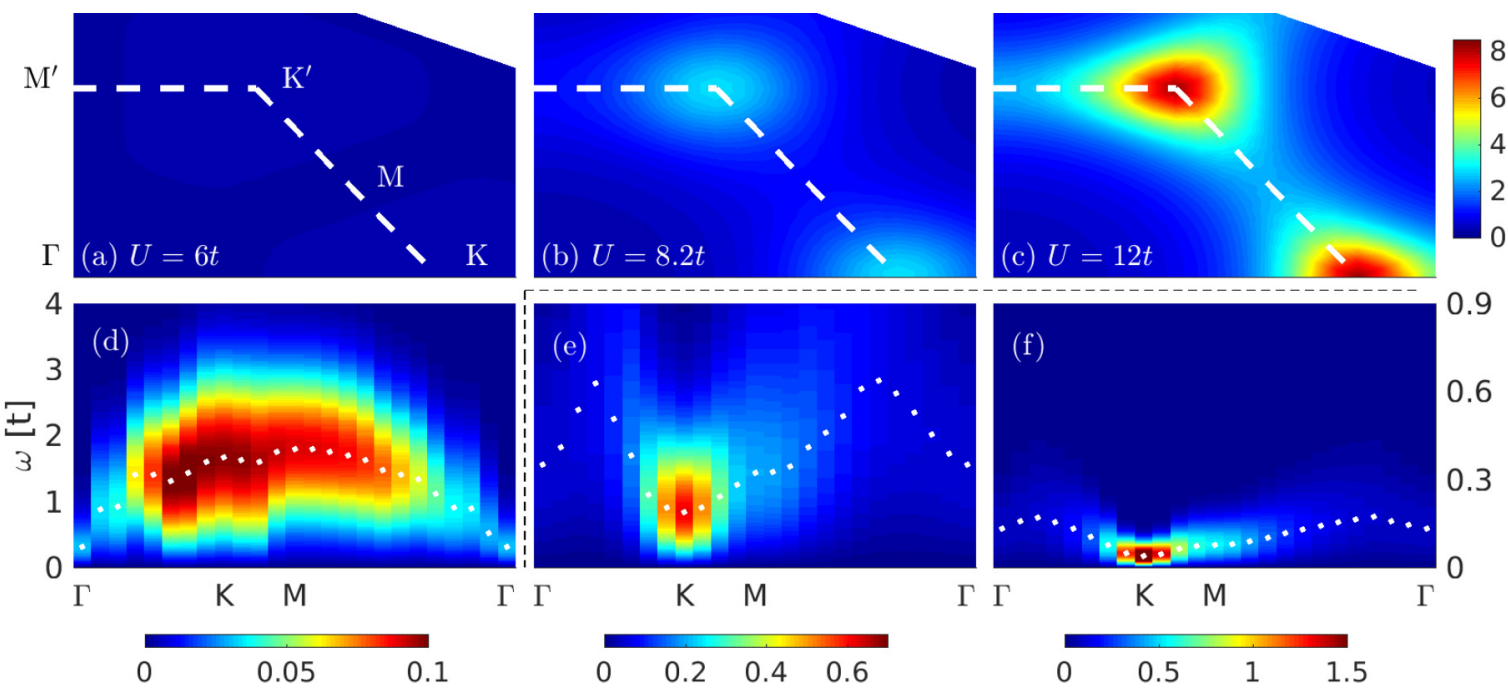

FIG. 5. (a)-(c) Static magnetic susceptibility in the momentum space $\chi_{m}\left(\mathbf{q}, i v_{0}\right)$ for $U=6 t, 8.2 t$, and $12 t$ at $T=t / 6$. The white dashed line delineates the Brillouin zone boundary. (d)-(f) Imaginary part of the dynamic magnetic susceptibility $\operatorname{Im} \chi_{m}(\mathbf{q}, \omega)$ along the high-symmetry direction for the same values of $U$. White dots: Peak position of the spectra $\omega_{m}(\mathbf{q})$.

Figures 5(d)-5(f) show the imaginary part of the dynamical magnetic susceptibility $\operatorname{Im} \chi_{m}(\mathbf{q}, \omega)$ as a function of energy $\omega$ and momentum $\mathbf{q}$ along a high-symmetry path in the Brillouin zone [see labels in Fig. 5(a)]. White dots show the energy $\omega_{m}(\mathbf{q})$ of the maximum intensity at each momentum, referred to as the spin-wave dispersion. It is clear that, except for momenta near the $\Gamma$ point, the intensity of spin excitations is enhanced and the spin excitation energy decreases as $U$ increases. At $U=6 t$ there is no dominant spin excitation and spin fluctuations occur in a large part of the Brillouin zone. At $U=8.2 t$ and $U=12 t$ the spin excitation energy at the $K$ point is smaller compared to other momenta, and most spin fluctuations occur at the $K$ point. For $U=8.2 t$ and $U=12 t$, the spin excitation energy at the $\Gamma$ point is nonzero, violating the total spin conservation. This is an artifact of the LDFA [51,59], which is not a conserving approximation.

To understand the behavior of spin excitations at the $K$ point, where magnetic order forms at zero temperature, Fig. 6(a) shows the spin excitation energy $\omega_{m}(K)$ as a function of $U . \omega_{m}(K)$ approaches to two different values at small and large $U$, and a sharp decrease occurs as $U$ increases from $6 t$ to $7 t$. Figure 6(b) shows $\operatorname{Im} \chi_{m}(\mathbf{q}, \omega)$ at $\mathbf{q}=K$ for various different $U$ values. The maximum value of $\operatorname{Im} \chi_{m}(K, \omega)$ increases very little as $U$ increases from $6 t$ to $7 t$, while it increases rapidly as $U$ continues to increase. These results suggest that spin fluctuations start to condense at the $K$ point around $U=$ $7 t$. The density of states (DOS) at the Fermi surface, plotted in Fig. 6(a), shows that the system is still metallic at $U=7 t$. Our results therefore suggest that the strong spin fluctuations at the $K$ point not only exist in the insulating phase but also extend into the metallic phase.

This persistence of spin fluctuations can also be observed in the spin-lattice relaxation rate. Figure 7(a) plots the spinlattice relaxation rate $\left(T_{1} T\right)^{-1}$ as a function of temperature for the three values of $U .\left(T_{1} T\right)^{-1}$ is calculated via $\lim _{\omega \rightarrow 0} \sum_{\mathbf{q}} \frac{\operatorname{Im} \chi_{m}(\mathbf{q}, \omega)}{\omega}[60]$. It is shown that $\left(T_{1} T\right)^{-1}$ is enhanced as $U$ increases. At $U=12 t,\left(T_{1} T\right)^{-1}$ increases rapidly as temperature decreases, indicating the formation of a magnetic order at low temperature with the transition temperature $T \approx 0.125 t$. For $U=8.2 t,\left(T_{1} T\right)^{-1}$ increases very slowly at low temperature, consistent with the previous result that the magnetic order is absent at low temperature [56]. The increase of $\left(T_{1} T\right)^{-1}$ implies that spin fluctuations are not negligible. At $U=6 t,\left(T_{1} T\right)^{-1}$ is almost independent of temperature, consistent with the weak spin fluctuations shown in Fig. 5(d).

Figure 7(b) shows the spin-lattice relaxation rate of $\kappa$-(ET) $)_{2} \mathrm{Cu}_{2}(\mathrm{CN})_{3}$ measured under different pressures, extracted from Ref. [2]. The $x$ axis has been rescaled by $t$, which is about $0.055 \mathrm{eV}$ for $\kappa-(\mathrm{ET})_{2} \mathrm{Cu}_{2}(\mathrm{CN})_{3}$ [61]. At $0 \mathrm{GPa}$, corresponding to the spin-liquid region, $\left(T_{1} T\right)^{-1}$ monotonously increases as temperature decreases. At $0.4 \mathrm{GPa}$, corresponding to the metallic phase near the phase boundary, $\left(T_{1} T\right)^{-1}$ increases as $T$ decreases and reaches the maximum value

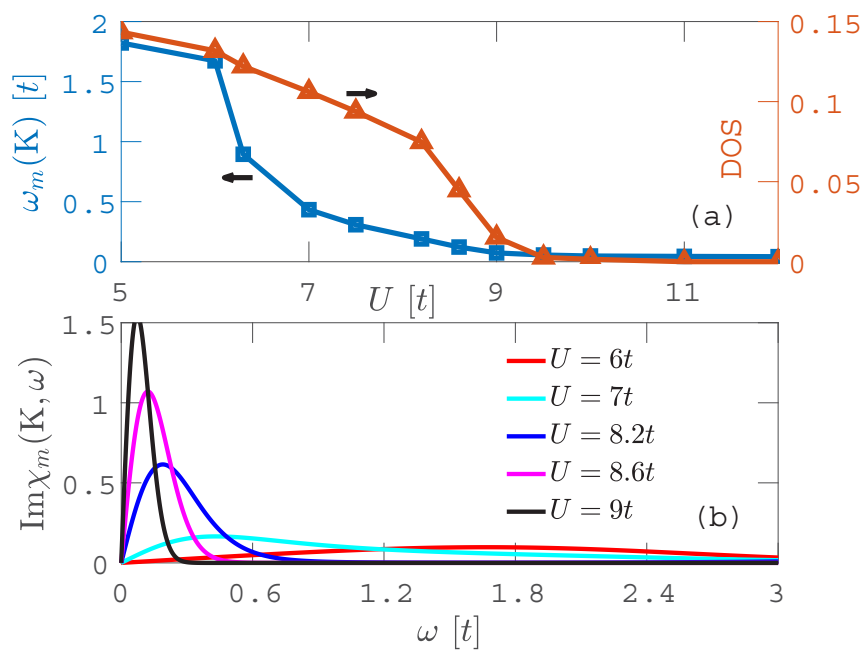

FIG. 6. (a) The spin excitation energy $\omega_{m}(K)$ at the $K$ point and the density of states (DOS) at $\omega=0$ as a function of $U$. (b) Imaginary part of dynamical magnetic susceptibility $\operatorname{Im} \chi_{m}(\mathbf{q}, \omega)$ at $\mathbf{q}=K$ as a function of $\omega$ for various different $U$. 


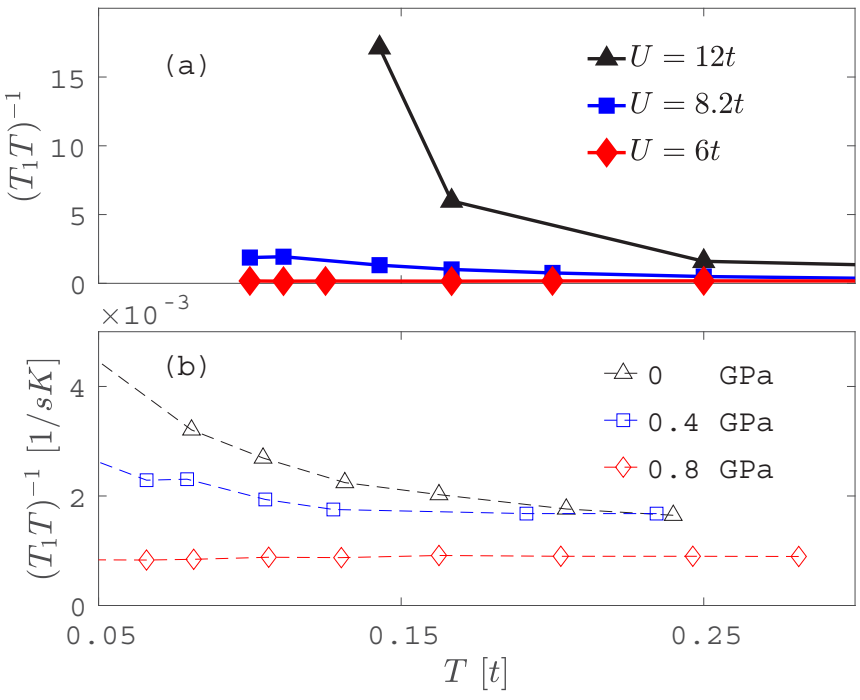

FIG. 7. (a) Spin-lattice decay rate $\left(T_{1} T\right)^{-1}$ as a function of temperature $T / t$ for different $U$ values. (b) shows $\left(T_{1} T\right)^{-1}$ from ${ }^{1} \mathrm{H}$ NMR measurements of $\kappa-(\mathrm{ET})_{2} \mathrm{Cu}_{2}(\mathrm{CN})_{3}$ [2]. The hopping integral $t$ for $\kappa-(\mathrm{ET})_{2} \mathrm{Cu}_{2}(\mathrm{CN})_{3}$ is about $0.055 \mathrm{eV}$ [61].

at about $0.03 t$. Continuing to decrease temperature, $\left(T_{1} T\right)^{-1}$ decreases due to the appearance of a superconducting state, which is not plotted in Fig. 7(b) and absent in our calculations. At $0.8 \mathrm{GPa},\left(T_{1} T\right)^{-1}$ is independent of temperature. We notice that the temperature-dependent behavior of $\left(T_{1} T\right)^{-1}$ for these three pressures is similar to that for the three values of $U$ we calculated. The main difference is the behavior of $\left(T_{1} T\right)^{-1}$ at high temperature. In experiment, $\left(T_{1} T\right)^{-1}$ has a smaller value at $0.8 \mathrm{GPa}$ than that for 0 and $0.4 \mathrm{GPa}$ at high temperature. In our calculations, $\left(T_{1} T\right)^{-1}$ approaches the same value at high temperature. This difference may be due to pressure changes of the lattice geometry in $\kappa-(\mathrm{ET})_{2} \mathrm{Cu}_{2}(\mathrm{CN})_{3}$ [62].

Leaving these differences aside, both our simulated and experimental spin-lattice relaxation rates imply that spin fluctuations persist into the metallic phase, which is consistent with previous finite-temperature Lanczos studies [15]. Moreover, our dynamical momentum-dependent magnetic susceptibilities further explain the behaviors of spin excitations at each momentum and provide a comprehensive understanding of spin behaviors in correlated metals.

\section{Charge susceptibility}

We next examine the charge properties. Figures 8(a)8 (c) show the static charge susceptibility $\chi_{c}\left(\mathbf{q}, i v_{0}\right)$ for the same three values of $U$. It is clearly seen that $\chi_{c}\left(\mathbf{q}, i v_{0}\right)$ is suppressed as $U$ increases and is invisible in the insulator $(U=12 t)$. At $U=6 t$ the maximum value of $\chi_{c}\left(\mathbf{q}, i v_{0}\right)$ is located at the $\Gamma$ point, indicating a uniform charge distribution. $\chi_{c}\left(\mathbf{q}, i v_{0}\right)$ along the $\Gamma \rightarrow \Gamma^{\prime}$ direction $\left(\Gamma^{\prime}\right.$ is the $\Gamma$ point in the second Brillouin zone) is larger compared to the other momenta. These features are weaker at $U=8.2$ and invisible at $U=12 t$.

Figures 8(d)-8(f) plot the imaginary part of the charge susceptibility $\operatorname{Im} \chi_{c}(\mathbf{q}, \omega)$ for these three values of $U$. At $U=6 t$ there is no charge gap at the $\Gamma$ point and the maximum energy of the charge excitation is located around the Brillouin zone boundary, corresponding to the charge excitation from the bottom to the top of the band. Little change is visible near the crossover, $U=8.2 t$. The nonzero charge excitation at the $\Gamma$ point is because LDFA violates the total charge conservation [51,59]. We also note that the maximum energy of the charge excitation does not change much as $U$ increases before the Mott gap is opened, while it increases rapidly as the gap is opened. Our predicted charge spectra may be observed in momentum-resolved electron-loss spectroscopy measurements on triangular compounds.

Finally, we compare our magnetic and charge susceptibilities to the bare susceptibility $\operatorname{Im} \chi_{0}(\mathbf{q}, \omega)$ [63], which is evaluated by a multiplication of two Green's functions [63]. The low-energy spectra of $\operatorname{Im} \chi_{0}(\mathbf{q}, \omega)$ and $\operatorname{Im} \chi_{m}(\mathbf{q}, \omega)$ are consistent at $U=6 t$ but inconsistent at $U=8.2$ and $U=12 t$. The high-energy spectra of $\operatorname{Im} \chi_{0}(\mathbf{q}, \omega)$ are consistent with $\operatorname{Im} \chi_{c}(\mathbf{q}, \omega)$ only near the Brillouin zone boundary for these three values of $U$. These discrepancies suggest that vertex corrections are essential.
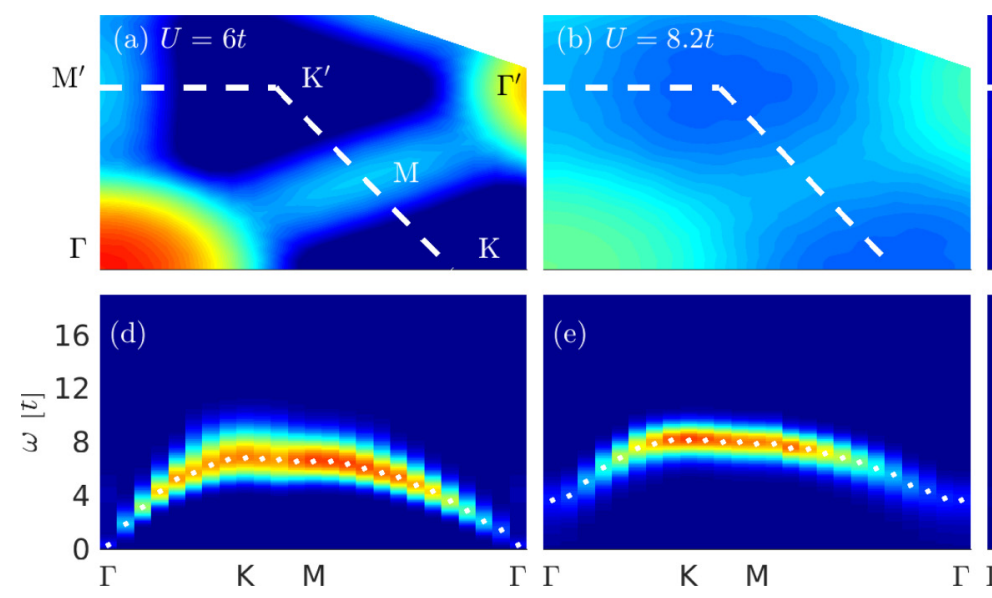
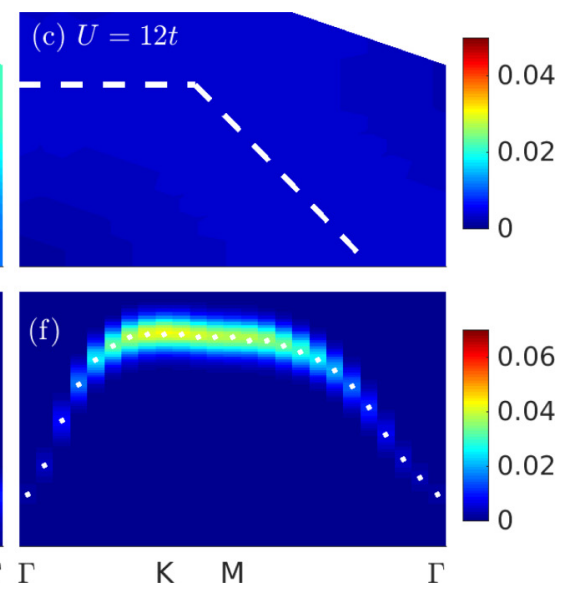

FIG. 8. (a)-(c) Static charge susceptibility in momentum space $\chi_{c}\left(\mathbf{q}, i v_{0}\right)$ for $U=6 t, 8.2 t$, and $12 t$ at $T=t / 6$. The white dashed line delineates the Brillouin zone boundary. (d)-(f) Imaginary part of the dynamical charge susceptibility $\operatorname{Im} \chi_{c}(\mathbf{q}, \omega)$ along the high-symmetry direction for the same three values of $U$. White dots: Peak position of the spectra. 


\section{CONCLUSION AND DISCUSSION}

In conclusion, we have studied the crossover from metal to insulator in a triangular Hubbard model using the LDFA approach. We examined the momentum and energy dependence of the spin and charge spectra in the metallic, Mott insulating, and crossover regimes. In the insulator, we find most of low-energy magnetic excitations exist at the $K$ point, corresponding to the $120^{\circ}$ antiferromagnetic order. These magnetic excitations persist into the crossover and metallic regions and move to higher energy as $U \leqslant 6 t$. Our results indicate that the strong spin fluctuations at the $K$ point exist not only in the insulator but also the metallic phase. This conclusion is further corroborated by our calculated spin-lattice relaxation times and NMR measurements of $\kappa-(\mathrm{ET})_{2} \mathrm{Cu}_{2}(\mathrm{CN})_{3}$.

Our results explain the momentum-dependent spin and charge properties of the Hubbard model in the triangular system. Usually, the spin properties of the triangular system are obtained by studying Heisenberg models, which cannot describe properties of an itinerant system. In contrast, here, magnetic properties of both metallic and insulating phases are obtained on an equal footing.

Our results show that magnetic excitations at the $K$ point persist deep into the metallic region at high temperature. At low temperature, the metallic phase near the phase boundary becomes superconducting in $\kappa$-(BEDT-TTF $)_{2} \mathrm{Cu}_{2}(\mathrm{CN})_{3}$. It would be interesting to experimentally study magnetic excitations at low temperature and in the superconducting phase in this material.

\section{ACKNOWLEDGMENTS}

This work was supported by the National Science Foundation (NSF) under Grant No. DMR-1606348. This work used resources of the Extreme Science and Engineering Discovering Enviroment (XSEDE) under Grant No. TG-DMR130036.
[1] Y. Shimizu, K. Miyagawa, K. Kanoda, M. Maesato, and G. Saito, Phys. Rev. Lett. 91, 107001 (2003).

[2] Y. Kurosaki, Y. Shimizu, K. Miyagawa, K. Kanoda, and G. Saito, Phys. Rev. Lett. 95, 177001 (2005).

[3] T. Itou, A. Oyamada, S. Maegawa, M. Tamura, and R. Kato, J. Phys.: Condens. Matter 19, 145247 (2007).

[4] M. Yamashita, N. Nakata, Y. Senshu, M. Nagata, H. M. Yamamoto, R. Kato, T. Shibauchi, and Y. Matsuda, Science 328, 1246 (2010).

[5] S. Yamashita, T. Yamamoto, Y. Nakazawa, M. Tamura, and R. Kato, Nat. Commun. 2, 275 (2011).

[6] T. Isono, H. Kamo, A. Ueda, K. Takahashi, M. Kimata, H. Tajima, S. Tsuchiya, T. Terashima, S. Uji, and H. Mori, Phys. Rev. Lett. 112, 177201 (2014).

[7] Y. Zhou, K. Kanoda, and T.-K. Ng, Rev. Mod. Phys. 89, 025003 (2017).

[8] T. Shirakawa, T. Tohyama, J. Kokalj, S. Sota, and S. Yunoki, Phys. Rev. B 96, 205130 (2017).

[9] R. Rawl, L. Ge, H. Agrawal, Y. Kamiya, C. R. Dela Cruz, N. P. Butch, X. F. Sun, M. Lee, E. S. Choi, J. Oitmaa, C. D. Batista, M. Mourigal, H. D. Zhou, and J. Ma, Phys. Rev. B 95, 060412(R) (2017).

[10] A. Pustogow, M. Bories, A. Löhle, R. Rösslhuber, E. Zhukova, B. Gorshunov, S. Tomić, J. A. Schlueter, R. Hübner, T. Hiramatsu, Y. Yoshida, G. Saito, R. Kato, T. H. Lee, V. Dobrosavljević, S. Fratini, and M. Dressel, Nat. Mater. 17, 773 (2018).

[11] S. Rachel, M. Laubach, J. Reuther, and R. Thomale, Phys. Rev. Lett. 114, 167201 (2015).

[12] F. Adler, S. Rachel, M. Laubach, J. Maklar, A. Fleszar, J. Schäfer, and R. Claessen, Phys. Rev. Lett. 123, 086401 (2019).

[13] T. Koretsune, Y. Motome, and A. Furusaki, J. Phys. Soc. Jpn. 76, 074719 (2007).

[14] R. T. Clay, H. Li, and S. Mazumdar, Phys. Rev. Lett. 101, 166403 (2008).

[15] J. Kokalj and R. H. McKenzie, Phys. Rev. Lett. 110, 206402 (2013).
[16] T. Watanabe, H. Yokoyama, Y. Tanaka, and J.-i. Inoue, J. Phys. Soc. Jpn. 75, 074707 (2006).

[17] T. Watanabe, H. Yokoyama, Y. Tanaka, and J. Inoue, Phys. Rev. B 77, 214505 (2008).

[18] L. F. Tocchio, C. Gros, R. Valentí, and F. Becca, Phys. Rev. B 89, 235107 (2014).

[19] L. F. Tocchio, H. Feldner, F. Becca, R. Valentí, and C. Gros, Phys. Rev. B 87, 035143 (2013).

[20] P. Sahebsara and D. Sénéchal, Phys. Rev. Lett. 100, 136402 (2008).

[21] A. Yamada, Phys. Rev. B 89, 195108 (2014).

[22] K. Misumi, T. Kaneko, and Y. Ohta, Phys. Rev. B 95, 075124 (2017).

[23] H.-Y. Yang, A. M. Läuchli, F. Mila, and K. P. Schmidt, Phys. Rev. Lett. 105, 267204 (2010).

[24] H. Morita, S. Watanabe, and M. Imada, J. Phys. Soc. Jpn. 71 , 2109 (2002).

[25] B. Kyung and A.-M. S. Tremblay, Phys. Rev. Lett. 97, 046402 (2006).

[26] T. Ohashi, T. Momoi, H. Tsunetsugu, and N. Kawakami, Phys. Rev. Lett. 100, 076402 (2008).

[27] A. Liebsch, H. Ishida, and J. Merino, Phys. Rev. B 79, 195108 (2009).

[28] D. Galanakis, T. D. Stanescu, and P. Phillips, Phys. Rev. B 79, 115116 (2009).

[29] T. Sato, K. Hattori, and H. Tsunetsugu, Phys. Rev. B 86, 235137 (2012).

[30] H. Lee, G. Li, and H. Monien, Phys. Rev. B 78, 205117 (2008).

[31] H. T. Dang, X. Y. Xu, K.-S. Chen, Z. Y. Meng, and S. Wessel, Phys. Rev. B 91, 155101 (2015).

[32] Q. Q. Ge, H. C. Xu, X. P. Shen, M. Xia, B. P. Xie, F. Chen, Y. Zhang, R. Kato, T. Tsumuraya, T. Miyazaki, M. Matsunami, S. Kimura, and D. L. Feng, Phys. Rev. B 89, 075105 (2014).

[33] P. Lemmens, K. Y. Choi, V. Gnezdilov, E. Y. Sherman, D. P. Chen, C. T. Lin, F. C. Chou, and B. Keimer, Phys. Rev. Lett. 96, 167204 (2006). 
[34] Y. Shimizu, T. Hiramatsu, M. Maesato, A. Otsuka, H. Yamochi, A. Ono, M. Itoh, M. Yoshida, M. Takigawa, Y. Yoshida, and G. Saito, Phys. Rev. Lett. 117, 107203 (2016).

[35] S. Ito, N. Kurita, H. Tanaka, S. Ohira-Kawamura, K. Nakajima, S. Itoh, K. Kuwahara, and K. Kakurai, Nat. Commun. 8, 235 (2017).

[36] V. I. Iglovikov, E. Khatami, and R. T. Scalettar, Phys. Rev. B 92, 045110 (2015).

[37] A. N. Rubtsov, M. I. Katsnelson, and A. I. Lichtenstein, Phys. Rev. B 77, 033101 (2008).

[38] S. Brener, H. Hafermann, A. N. Rubtsov, M. I. Katsnelson, and A. I. Lichtenstein, Phys. Rev. B 77, 195105 (2008).

[39] G. Li, H. Lee, and H. Monien, Phys. Rev. B 78, 195105 (2008).

[40] H. Hafermann, F. Lechermann, A. N. Rubtsov, M. I. Katsnelson, A. Georges, and A. I. Lichtenstein, Strong electronic correlations: Dynamical mean-field theory and beyond, in Modern Theories of Many-Particle Systems in Condensed Matter Physics, edited by D. C. Cabra, A. Honecker, and P. Pujol (Springer, Berlin, 2012), pp. 145-214.

[41] A. E. Antipov, E. Gull, and S. Kirchner, Phys. Rev. Lett. 112, 226401 (2014).

[42] G. Rohringer, H. Hafermann, A. Toschi, A. A. Katanin, A. E. Antipov, M. I. Katsnelson, A. I. Lichtenstein, A. N. Rubtsov, and K. Held, Rev. Mod. Phys. 90, 025003 (2018).

[43] A. E. Antipov, J. P. LeBlanc, and E. Gull, Phys. Procedia 68, 43 (2015).

[44] A. Georges, G. Kotliar, W. Krauth, and M. J. Rozenberg, Rev. Mod. Phys. 68, 13 (1996).

[45] E. Gull, A. J. Millis, A. I. Lichtenstein, A. N. Rubtsov, M. Troyer, and P. Werner, Rev. Mod. Phys. 83, 349 (2011).

[46] E. Gull, P. Werner, O. Parcollet, and M. Troyer, Europhys. Lett. 82, 57003 (2008).

[47] E. Gull, P. Staar, S. Fuchs, P. Nukala, M. S. Summers, T. Pruschke, T. C. Schulthess, and T. Maier, Phys. Rev. B 83, 075122 (2011).

[48] J. P. F. LeBlanc, A. E. Antipov, F. Becca, I. W. Bulik, G. K.-L. Chan, C.-M. Chung, Y. Deng, M. Ferrero, T. M. Henderson, C. A. Jiménez-Hoyos, E. Kozik, X.-W. Liu, A. J. Millis, N. V.
Prokof'ev, M. Qin, G. E. Scuseria, H. Shi, B. V. Svistunov, L. F. Tocchio, I. S. Tupitsyn, S. R. White, S. Zhang, B.-X. Zheng, Z. Zhu, and E. Gull (Simons Collaboration on the Many-Electron Problem), Phys. Rev. X 5, 041041 (2015).

[49] T. Ribic, P. Gunacker, S. Iskakov, M. Wallerberger, G. Rohringer, A. N. Rubtsov, E. Gull, and K. Held, Phys. Rev. B 96, 235127 (2017).

[50] S. Iskakov, A. E. Antipov, and E. Gull, Phys. Rev. B 94, 035102 (2016).

[51] J. P. F. LeBlanc, S. Li, X. Chen, R. Levy, A. E. Antipov, A. J. Millis, and E. Gull, Phys. Rev. B 100, 075123 (2019).

[52] T. A. Maier, M. Jarrell, T. Pruschke, and M. H. Hettler, Rev. Mod. Phys. 77, 1027 (2005).

[53] R. Levy, J. LeBlanc, and E. Gull, Comput. Phys. Commun. 215, 149 (2017).

[54] A. Gaenko, A. Antipov, G. Carcassi, T. Chen, X. Chen, Q. Dong, L. Gamper, J. Gukelberger, R. Igarashi, S. Iskakov, M. Könz, J. LeBlanc, R. Levy, P. Ma, J. Paki, H. Shinaoka, S. Todo, M. Troyer, and E. Gull, Comput. Phys. Commun. 213, 235 (2017).

[55] M. Jarrell and J. Gubernatis, Phys. Rep. 269, 133 (1996).

[56] G. Li, A. E. Antipov, A. N. Rubtsov, S. Kirchner, and W. Hanke, Phys. Rev. B 89, 161118(R) (2014).

[57] M. Laubach, R. Thomale, C. Platt, W. Hanke, and G. Li, Phys. Rev. B 91, 245125 (2015).

[58] T. Yoshioka, A. Koga, and N. Kawakami, Phys. Rev. Lett. 103, 036401 (2009).

[59] H. Hafermann, E. G. C. P. van Loon, M. I. Katsnelson, A. I. Lichtenstein, and O. Parcollet, Phys. Rev. B 90, 235105 (2014).

[60] X. Chen, J. P. F. LeBlanc, and E. Gull, Nat. Commun. 8, 14986 (2017).

[61] K. Nakamura, Y. Yoshimoto, T. Kosugi, R. Arita, and M. Imada, J. Phys. Soc. Jpn. 78, 083710 (2009).

[62] R. T. Clay, N. Gomes, and S. Mazumdar, Phys. Rev. B 100, 115158 (2019).

[63] See Supplemental Material at http://link.aps.org/supplemental/ 10.1103/PhysRevResearch.2.013295 for a comparison of the spin-lattice decay rate from DMFT and LDFA calculations, and the bare susceptibility are shown. 